\title{
Spinal Nitric Oxide Synthase Type II Increases Neurosteroid-metabolizing Cytochrome P450c17 Expression in a Rodent Model of Neuropathic Pain
}

\author{
Sheu-Ran Choi ${ }^{1}$, Alvin J Beitz ${ }^{2}$ and Jang-Hern Lee ${ }^{1 *}$ \\ ${ }^{1}$ Department of Veterinary Physiology, BK21 PLUS Program for Creative Veterinary Science Research, Research Institute \\ for Veterinary Science and College of Veterinary Medicine, Seoul National University, Seoul 08826, Korea, ${ }^{2}$ Department of \\ Veterinary and Biomedical Sciences, College of Veterinary Medicine, University of Minnesota, St. Paul, MN 55108, USA
}

\begin{abstract}
We have previously demonstrated that the neurosteroid dehydroepiandrosterone sulfate (DHEAS) induces functional potentiation of $N$-methyl-D-aspartate (NMDA) receptors via increases in phosphorylation of NMDA receptor GluN1 subunit (pGluN1). However, the modulatory mechanisms responsible for the expression of the DHEA-synthesizing enzyme, cytochrome P450c17 following peripheral nerve injury have yet to be examined. Here we determined whether oxidative stress induced by the spinal activation of nitric oxide synthase type II (NOS-II) modulates the expression of P450c17 and whether this process contributes to the development of neuropathic pain in rats. Chronic constriction injury (CCI) of the sciatic nerve induced a significant increase in the expression of NOS-II in microglial cells and NO levels in the lumbar spinal cord dorsal horn at postoperative day 5. Intrathecal administration of the NOS-II inhibitor, L-NIL during the induction phase of neuropathic pain (postoperative days $0 \sim 5$ ) significantly reduced the CCI-induced development of mechanical allodynia and thermal hyperalgesia. Sciatic nerve injury increased the expression of PKCand PKA-dependent pGluN1 as well as the mRNA and protein levels of P450c17 in the spinal cord at postoperative day 5, and these increases were suppressed by repeated administration of L-NIL. Co-administration of DHEAS together with L-NIL restored the development of neuropathic pain and pGluN1 that were originally inhibited by L-NIL administration alone. Collectively these results provide strong support for the hypothesis that activation of NOS-II increases the mRNA and protein levels of P450c17 in the spinal cord, ultimately leading to the development of central sensitization and neuropathic pain induced by peripheral nerve injury.
\end{abstract}

Key words: Nitric oxide synthase type II, Cytochrome P450c17, Dehydroepiandrosterone sulfate, Phosphorylation, Neuropathic pain

\section{INTRODUCTION}

Several lines of evidence indicate that some steroids, called

Received May 4,2019, Revised June 7,2019,

Accepted July 8, 2019

* To whom correspondence should be addressed.

TEL: 82-2-880-1272, FAX: 82-2-885-2732

e-mail:jhl1101@snu.ac.kr neurosteroids, are synthesized in the nervous system in an independent manner from the steroidogenic organs and profoundly influence neuronal excitability $[1,2]$. One of these neurosteroids, dehydroepiandrosterone sulfate (DHEAS), has been found within the central nervous system of several mammalian species [3,4]. DHEAS is a negative modulator of the gamma-aminobutyric acid type $\mathrm{A}\left(\mathrm{GABA}_{\AA}\right)$ receptors and a positive activator of both sigma-1 receptors and $N$-methyl-D-aspartate (NMDA) receptors in the central nervous system, thus acting as an excitatory neurosteroid 
[5]. In this regard DHEAS enhances long term potentiation in rat hippocampal slices [6] and also improves memory and learning [7]. In a previous study, we demonstrated that intrathecal administration of DHEAS significantly enhances NMDA-induced nociceptive behaviors via increases in protein kinase $\mathrm{C}$ (PKC)- and protein kinase A (PKA)-dependent phosphorylation of the NMDA receptor GluN1 subunit (pGluN1), which serves as a critical contributor to central sensitization [8]. Although we have recently suggested that the dehydroepiandrosterone (DHEA)-synthesizing enzyme, cytochrome P450c17 (steroid 17-alpha-hydroxylase/17,20-lyase) is upregulated in the spinal cord during the induction phase of peripheral neuropathy following chronic constriction injury (CCI) of the sciatic nerve and its activation plays an important role in the development of chronic neuropathic pain [9], there is limited understanding of the mechanisms underlying the modulation of P450c17 expression during the induction phase of neuropathic pain.

Based on data from experimental studies, there is increasing support for the involvement of both peripheral and central mechanisms in chronic neuropathic pain. Nitric oxide $(\mathrm{NO})$ is a free gaseous signaling molecule synthesized by different isoforms of nitric oxide synthase (NOS) and has been linked with the development and maintenance of neuropathic pain [10-13]. Among the isoforms of NOS, nitric oxide synthase type II (NOS-II), which is also known as inducible NOS, has been demonstrated to produce a large amount of NO, thus, its increased expression plays an important role in the facilitation of inflammatory reactions and neurotoxicity $[12,14]$. It has been suggested that local activation of the NOS-II/NO system plays an important role in the pathogenesis of peripheral neuropathy and the development of chronic pain [15]. In the peripheral nervous system, NOS-II was detected locally in the paw $6 \mathrm{~h}$ after Complete Freund's Adjuvant (CFA) injection. Inhibition of iNOS significantly reversed CFA-induced hypersensitivity to pain and edema in a dose-dependent manner demonstrating a role for peripherally-expressed NOS-II in pain conditions with an inflammatory component [16]. Although NOS-II regulates the gene expression of a variety of proteins in the central nervous system, resulting in modulation of the synaptic environment and neuronal excitability in the spinal cord and brain [14], the potential role of NOS-II activation on neurosteroid signaling induced by $\mathrm{P} 450 \mathrm{c} 17$ is poorly understood, particularly in the case of spinal nociceptive signaling transmission.

The main purpose of this study was to investigate whether spinal NOS-II modulates the expression of P450c17 and GluN1 phosphorylation, ultimately leading to the development of chronic pain in a rat model of neuropathic pain. In this regard, we examined whether: (1) sciatic nerve injury increases NOS-II expression and
NO levels in the lumbar spinal cord dorsal horn of CCI rats; (2) inhibition of spinal NOS-II with L-NIL suppresses the development of neuropathic pain, GluN1 phosphorylation, and spinal P450c17 expression; and (3) co-administration of DHEAS together with LNIL restores the CCI-induced development of neuropathic pain and GluN1 phosphorylation that were significantly reduced by LNIL administration alone.

\section{MATERIALS AND METHODS}

\section{Experimental animals and surgery}

Male Sprague-Dawley rats (180 200 g) were purchased from the Laboratory Animal Center of Seoul National University (Seoul, Republic of Korea). Animals had free access to food and water and were kept in temperature and light controlled rooms $\left(23 \pm 2^{\circ} \mathrm{C}\right.$, $12 / 12 \mathrm{~h}$ light/dark cycle) for 3 days prior to the beginning of the experiment. The experimental protocols for animal usage were reviewed and approved by the SNU Institutional Animal Care and Use Committee and were consistent with the Guide for the Care and Use of Laboratory Animals published by the US National Institutes of Health (NIH publication No. 96-01, revised 1996).

A chronic constriction injury (CCI) of the common sciatic nerve was performed according to the method described by Bennett and Xie [17]. Briefly, rats were anesthetized with 3\% isoflurane in a mixture of $\mathrm{N}_{2} \mathrm{O} / \mathrm{O}_{2}$ gas. The right sciatic nerve was exposed and 4 loose ligatures of 4-0 chromic gut were placed around the nerve and spaced apart from each other by 1.0 - to $1.5-\mathrm{mm}$ separations. Sham surgery was performed by exposing the sciatic nerve in the same manner, but without ligating the nerve. After surgery, animals recovered in clear plastic cages at $27^{\circ} \mathrm{C}$ with a thick layer of sawdust bedding.

\section{Drugs and administration}

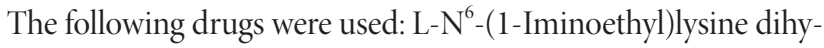
drochloride (L-NIL, an NOS-II inhibitor; 6, 20, 60 nmol); dehydroepiandrosterone sulfate (DHEAS; $3,10 \mathrm{nmol}$ ). L-NIL and DHEAS were purchased from Sigma-Aldrich (St. Louis, MO, USA). The doses of L-NIL were selected based on the results of preliminary experiments and the doses of DHEAS were selected based on the doses used in a previous study from our laboratories [8]. L-NIL was dissolved in physiological saline and DHEAS was dissolved in $1 \% \mathrm{DMSO}$ in physiological saline. The injection volume was 20 $\mu \mathrm{l}$. Drugs were administered intrathecally twice a day on postoperative days $0 \sim 5$, during the induction phase of neuropathic pain development.

Intrathecal drug administration was performed using a $50 \mu \mathrm{l}$ Hamilton syringe connected to a 27 -gauge needle as previously 
described [18]. Rats were briefly anesthetized with 3\% isoflurane in a mixture of $\mathrm{N}_{2} \mathrm{O} / \mathrm{O}_{2}$ gas to prevent any handling-induced stress and to allow more accurate injection of drugs. The rat was held tightly between the thumb and middle finger at the level of the both iliac crests, and the fifth lumbar spinous process was palpated with the index finger. The needle was inserted through the vertebral column into the $\mathrm{L}_{5-6}$ intervertebral space and successful insertion of the needle into the intrathecal space was determined by a tail flick response. Each drug was slowly injected over a 10 second period. Then, the needle was carefully removed from the spinal cord. The drug control groups received an identical injection of vehicle.

\section{Behavioral assessment}

Pain behavioral tests were performed on the ipsilateral (injured) hind paw 1 day before surgery on all animals to obtain normal baseline values of paw withdrawal responses to mechanical and thermal stimulation. Then, animals were randomly assigned to experimental and control groups. Animals were tested again at 1,3, $4,5,7,9,14,21$ and 28 days following CCI surgery. All behavioral analyses were performed blindly.

To assess nociceptive responses to innocuous mechanical stimuli (mechanical allodynia), we measured paw withdrawal response frequency (PWF) by using a von Frey filament with a force of 2.0 $\mathrm{g}$ (North Coast Medical, Morgan Hill, CA) as described in a previous study from our laboratories [18]. Rats were placed in acrylic chambers on a wire mesh floor and allowed to habituate before testing. A von Frey filament was applied to the plantar surface of each hind paw for a $3 \mathrm{sec}$ period before removal and we subsequently recorded whether there was a withdrawal of the hind limb to the filament. The filament was applied 10 times to the hind paw with a $10 \mathrm{sec}$ interval between each application. Then, the number of paw withdrawal responses was counted and the results of mechanical behavioral testing in the hind paw were expressed as a percent withdrawal response frequency, which represented the percentage of paw withdrawals out of the maximum of 10 .

To assess nociceptive responses to noxious heat stimuli (thermal hyperalgesia), we measured the paw withdrawal response latency (PWL, s) using a plantar analgesia meter (Model 390, IITC Life Science Inc., Woodland Hills, CA, USA) as previously described by Hargreaves et al. with minor modifications [19]. Rats were placed in acrylic cylinders on a glass floor and allowed to habituate before testing. A radiant heat source was positioned under the floor beneath the hind paw and then the withdrawal latency was measured. The test was duplicated in the ipsilateral hind paw, and the mean withdrawal latency was calculated. A cutoff latency in the absence of a response was set at $20 \mathrm{~s}$ to prevent tissue damage.

\section{Measurement of nitric oxide in the spinal cord}

Nitric oxide (NO) was determined in the spinal cord dorsal horns from the lumbar enlargement using a nitric oxide detection kit (cat\# ADI-917-020, Enzo Life sciences Inc., USA) as described previously [20]. This nitric oxide (total) detection kit is based on the enzymatic conversion of nitrate to nitrite by the enzyme nitrate reductase, followed by the Griess reaction to form a colored azo dye product. Advantages of this method include a strong literature background, numerous commercially available reagent kits and wide availability of infrastructure. Animals were deeply anesthetized with $3 \%$ isoflurane in a mixture of $\mathrm{N}_{2} \mathrm{O} / \mathrm{O}_{2}$ gas on day 5 postsurgery and perfused transcardially with calcium-free Tyrode's solution. The isolated spinal cords were homogenized in PBS by sonication. Homogenates were subsequently centrifuged at 400 g for $10 \mathrm{~min}$ at $4^{\circ} \mathrm{C}$ and, then, the supernatant was used for nitric oxide detection following the manufacturer's recommendation.

\section{Western blot assay}

The Western blot assay was performed as described previously [18]. Animals were deeply anesthetized with $3 \%$ isoflurane in a mixture of $\mathrm{N}_{2} \mathrm{O} / \mathrm{O}_{2}$ gas on day 5 post-surgery and perfused transcardially with calcium-free Tyrode's solution. The ipsilateral spinal cord dorsal horns from the lumbar enlargement were homogenized in lysis buffer (20 mM Tris-HCl, 10 mM EGTA, 2 mM EDTA, pH 7.4 and proteinase inhibitors) containing 1\% Triton $\mathrm{X}-100$. Homogenates were subsequently centrifuged at $15,000 \mathrm{rpm}$ for $40 \mathrm{~min}$ at $4^{\circ} \mathrm{C}$ and, then, the supernatant was used for Western blot analysis.

The protein concentration was estimated by the Bradford dye assay (Bio-Rad Laboratories). Spinal cord homogenates ( $25 \mu \mathrm{g}$ protein) were separated using 10\% SDS-polyacrylamide gel electrophoresis and transferred to nitrocellulose membrane. After the blots had been washed with TBST (10 mM Tris-HCl, pH 7.6, $150 \mathrm{mM} \mathrm{NaCl}$ and $0.05 \%$ Tween-20), the membranes were blocked with 5\% skimmed milk for $1 \mathrm{~h}$ at RT and incubated at $4^{\circ} \mathrm{C}$ overnight with a primary antibody specific for NOS-II (rabbit polyclonal anti-NOS2 antibody, 1:1,000, cat\# sc-651, Santa Cruz Biotechnology Inc.), PKC-dependent pGluN1 (rabbit polyclonal anti-pGluN1 Ser896 antibody, 1:1,000, cat\# ABN88, Millipore Co., USA; The antibody-specificity information available from Millipore Corporation states that this antibody recognizes GluN1 when phosphorylated at serine 896 and that the immunogen was a KLH-conjugated linear peptide corresponding to rat GluN1 phosphorylated at serine 896), PKA-dependent pGluN1 (rabbit polyclonal anti-pGluN1 Ser897 antibody, 1:1,000, cat\# ABN99, Millipore Co., USA; The antibody-specificity information available from Millipore Corporation states that this antibody recognizes 
GluN1 when phosphorylated at serine 897 and the immunogen was a KLH-conjugated linear peptide corresponding to rat GluN1 phosphorylated at serine 897), P450c17 (rabbit monoclonal anticytochrome P450 17A1 antibody, 1:1,000, cat\# ab125022, Abcam plc.) or $\beta$-actin (mouse monoclonal anti- $\beta$-actin antibody, 1:5,000, cat\# sc-47778, Santa Cruz Biotechnology Inc.). After washing with TBST, membranes were incubated for $4 \mathrm{~h}$ at $4{ }^{\circ} \mathrm{C}$ with horseradish peroxidase-conjugated anti-rabbit or anti-mouse antibody (1:10,000, Santa Cruz Biotechnology Inc.). The bands were visualized with enhanced chemiluminescence (Amersham Biosciences). The positive pixel area of specific bands was measured using ImageJ software (ImageJ 1.45s; National Institutes of Health, USA) and normalized against the corresponding $\beta$-actin loading control bands. The mean value of control groups was set at $100 \%$. Thus, the $\%$ change relative to the mean value of control groups was then calculated in each group.

\section{Quantitative real-time PCR}

Quantitative real-time PCR was performed as described previously [9]. Animals were deeply anesthetized with 3\% isoflurane in a mixture of $\mathrm{N}_{2} \mathrm{O} / \mathrm{O}_{2}$ gas on day 5 post-surgery and perfused transcardially with calcium-free Tyrode's solution. The ipsilateral spinal cord dorsal horns from the lumbar enlargement were homogenized in Buffer RL, and RNA samples were extracted using a commercial RNA extraction kit (cat\# 9767, TaKaRa, Otsu, Shiga, Japan). Then, $1 \mu \mathrm{g}$ of RNA was reverse-transcribed with a reverse transcription-PCR premix (cat\# 25081, iNtRON Biotechnology, Seongnam, Korea). Reverse transcription was performed for $1 \mathrm{~h}$ at $45^{\circ} \mathrm{C}$ followed by $5 \mathrm{~min}$ at $95^{\circ} \mathrm{C}$. The cDNA samples were amplified with the specific mRNA primers and a $\mathrm{TB}^{\mathrm{TM}}$ Green Premix Ex Taq $^{\text {TM }}$ (cat\# RR420A, TaKaRa). Quantitative real-time PCR was performed as follows: $10 \mathrm{~min}$ at $95^{\circ} \mathrm{C}$ for DNA polymerase activation and 50 cycles of $15 \mathrm{~s}$ at $94^{\circ} \mathrm{C}, 15 \mathrm{~s}$ at $58^{\circ} \mathrm{C}$, and $30 \mathrm{~s}$ at $72^{\circ} \mathrm{C}$. The identity and specificity of the amplified PCR product was validated by melting curve analysis. The relative expression of $\mathrm{P} 450 \mathrm{c} 17$ mRNA was quantified with double delta $\mathrm{Ct}$ analysis, and the data were normalized with the expression of GAPDH mRNA. The quantitative real-time PCR experiment was duplicated for each sample and the mean mRNA level was calculated. The sequences of the mRNA primers used in this study are described in Table 1.

\section{Immunohistochemistry}

Rats were deeply anesthetized with 3\% isoflurane in a mixture of $\mathrm{N}_{2} \mathrm{O} / \mathrm{O}_{2}$ gas at day 5 post-CCI surgery and perfused transcardially with calcium-free Tyrode's solution and subsequently with fixative containing $4 \%$ paraformaldehyde in $0.1 \mathrm{M}$ phosphate buffer ( $\mathrm{pH}$ 7.4). The spinal cords were collected after perfusion, post- fixed in the identical fixative for $2 \mathrm{~h}$ at RT and then placed in 30\% sucrose in PBS ( $\mathrm{pH} 7.4)$ at $4^{\circ} \mathrm{C}$. Serial transverse sections $(40 \mu \mathrm{m})$ of the $\mathrm{L}_{4-5}$ spinal cord were cut using a cryostat (Leica CM1520, Leica Biosystems, Germany). Spinal tissue sections were washed with PBS and incubated for 2 days at $4{ }^{\circ} \mathrm{C}$ with a primary antibody specific for NOS-II (rabbit polyclonal anti-NOS2 antibody, 1:1,000, cat\# sc-651, Santa Cruz Biotechnology Inc.) or Iba-1 (goat polyclonal anti-Iba-1 antibody, 1:500, cat\# ab5076, Abcam plc.). The primary antibodies were detected by incubating the tissue in Alexa Fluor $^{\circledR} 568$ donkey anti-rabbit antibody (1:400, Invitrogen) or Alexa Fluor ${ }^{\circledR} 488$ donkey anti-goat antibody (1:400, Life Technologies) for $90 \mathrm{~min}$ at RT. Tissue sections were mounted on slides and visualized with a confocal microscope (Nikon Eclipse TE2000-E, Nikon, Japan).

\section{Statistical analysis}

Data are expressed as the mean \pm SEM. Statistical analyses were performed using Prism 5.0 (Graph Pad Software, San Diego, USA). Repeated measures two-way ANOVA was performed to determine differences in the behavioral data and this was followed by a Bonferroni multiple comparison test for post-hoc analysis. One-way ANOVA was used to determine differences in the data obtained from the area under curve (\%) for the behavioral experiments, Western blot analysis and quantitative real-time PCR, and this was followed by a Newman-Keuls multiple comparison test for post-hoc analysis. Comparisons between 2 groups were analyzed by two-tailed Student's t-test. ANOVA values and degrees of freedom are shown in Supplementary Tables S1 and S2. p-values less than 0.05 were considered statistically significant.

\section{RESULTS}

Sciatic nerve injury increases the expression of NOS-II in microglial cells and NO levels in the lumbar spinal cord dorsal horn of CCI rats

To determine whether chronic constriction injury (CCI) of the sciatic nerve induces a significant change in the expression of NOS-II and NO levels in the lumbar spinal cord dorsal horn, we

Table 1. Sequences of primers used in RT-PCR and real-time PCR

\begin{tabular}{ccc}
\hline $\begin{array}{c}\text { Target } \\
\text { genes }\end{array}$ & \multicolumn{1}{c}{ Forward 5'-3' } & \multicolumn{1}{c}{ Reverse 5'-3' } \\
\hline P450c17 & $\begin{array}{c}\text { TTTTGGCCCAAGT- } \\
\text { CAAAGAC }\end{array}$ & $\begin{array}{c}\text { CCCTTCTTCACGAG- } \\
\text { CACTTC }\end{array}$ \\
GAPDH & $\begin{array}{c}\text { AACTTTGGCATTGTG- } \\
\text { ACACATTGGGGGTAG- } \\
\end{array}$ & $\begin{array}{c}\text { GAAGG } \\
\text { GAACA }\end{array}$ \\
\hline
\end{tabular}

RT-PCR, reverse transcription polymerase chain reaction. 
examined the expression of NOS-II using Western blot analysis and measured nitrate concentration using an NO detection kit (a diazotization assay). Sciatic nerve injury significantly increased the expression of NOS-II (Fig. 1A) and NO levels (Fig. 1B) in the lumbar spinal cord dorsal horn at day 5 post-surgery as compared to that of the sham group (Fig. $1 \mathrm{~A}$ and $1 \mathrm{~B} ;{ }^{*} \mathrm{p}<0.05 \mathrm{vs}$. Sham). In addition, the results of immunohistochemistry analysis show that sciatic nerve injury increased NOS-II immunostaining, which is co-localized in Iba-1-positive microglial cells (Fig. 1C).

A

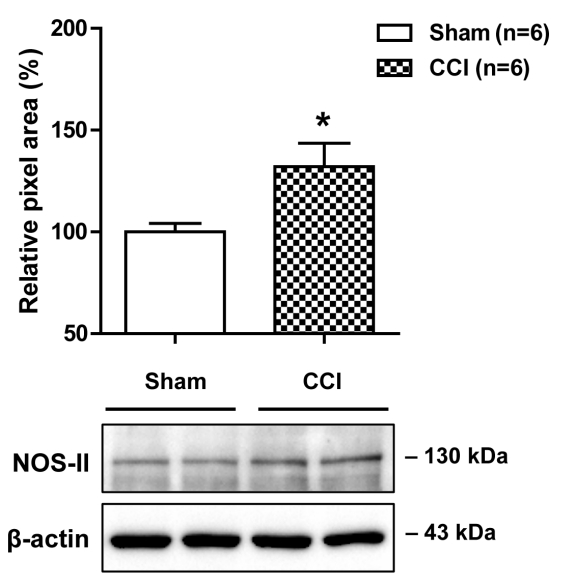

\section{I.t. administration of L-NIL suppresses the CCI-induced de-} velopment of neuropathic pain in rats

To verify whether the CCI-induced development of neuropathic pain is induced by activation of NOS-II, we intrathecally injected the NOS-II inhibitor, L-NIL during the induction phase of neuropathic pain. Sciatic nerve injury increased the paw withdrawal frequency (PWF, \%) to innocuous mechanical stimuli (mechanical allodynia) (Fig. $2 \mathrm{~A} ;{ }^{* * *} \mathrm{p}<0.001$ vs. Sham). The increase in PWF was significantly different at 4 days and this difference was sustained for 28 days post-surgery as compared to the sham group.

B

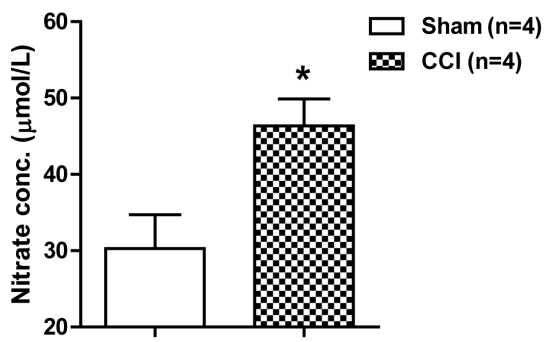

C
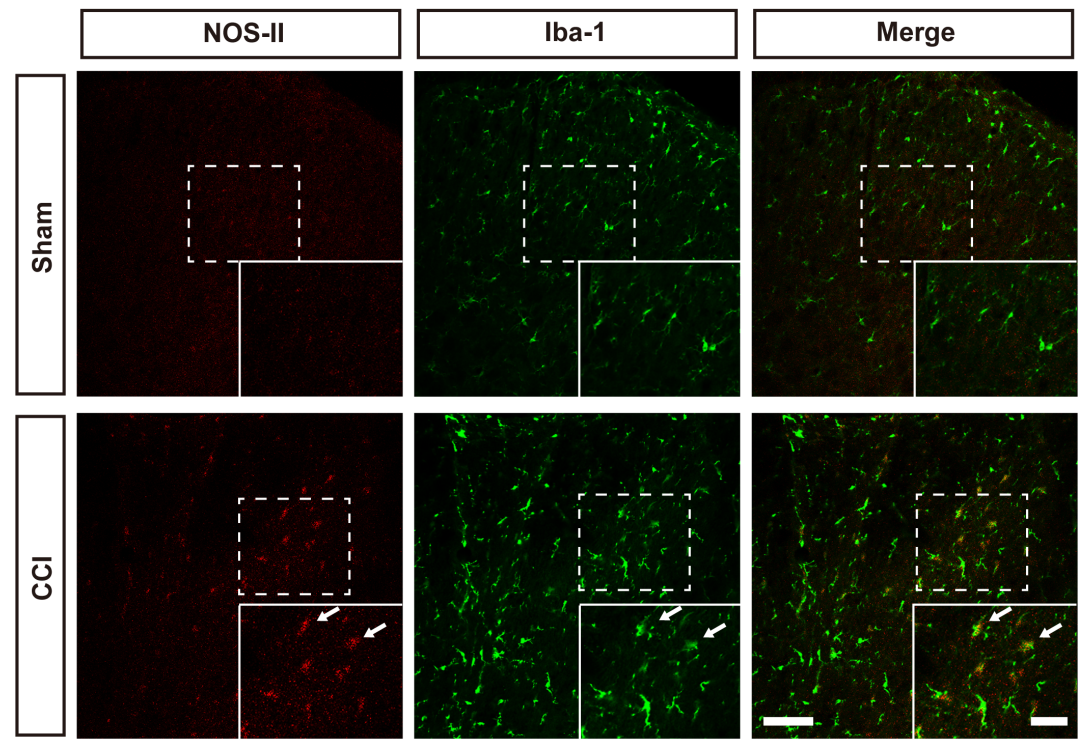

Fig. 1. Graphs illustrating the changes in expression of NOS-II and total NO production and NOS-II immunostaining in the lumbar spinal cord dorsal horn of CCI rats. (A) Results of Western blot analysis showed that sciatic nerve injury increased the expression of nitric oxide synthase type II (NOSII) in the spinal cord. $n=6$ rats/group. (B) Sciatic nerve injury increased total $\mathrm{NO}$ concentration (measured indirectly as the concentration of its stable decomposition product nitrate) in the spinal cord. $n=4$ rats/group. (C) Photomicrographs illustrate that NOS-II immunostaining (red) was increased in Iba-1-positive microglial cells (green) following CCI. Scale bar $=100 \mu \mathrm{m}$. Arrows in the magnified images depict examples of colocalization (Scale bar $=50$ $\mu \mathrm{m})$. The spinal cord dorsal horn was sampled at 5 days post-surgery. ${ }^{*} \mathrm{p}<0.05 \mathrm{vs}$. Sham. A and B, two-tailed Student's t-test. 
Repeated daily intrathecal (i.t.) administration of L-NIL (6, 20 and $60 \mathrm{nmol}$ ) during the induction phase of neuropathic pain (from days $0 \sim 5$ post-surgery) dose-dependently attenuated the CCIinduced development of mechanical allodynia as compared with

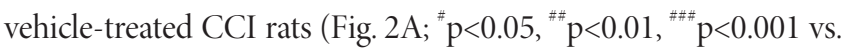
vehicle-treated group). In addition, area under curve (AUC, \%) data analysis showed a significant analgesic effect of L-NIL on the development of mechanical allodynia in neuropathic rats (Fig. 2B; ${ }^{* * *} \mathrm{p}<0.001$ vs. Sham, ${ }^{\# \#} \mathrm{p}<0.001$ vs. vehicle-treated group).

Sciatic nerve injury also decreased the paw withdrawal latency (PWL, s) to noxious heat stimulation (thermal hyperalgesia) as compared to the sham group, and this decrease was dosedependently inhibited by i.t. administration of L-NIL (6, 20 and 60 $\mathrm{nmol}$ ) during the induction phase of neuropathic pain (from days $0 \sim 5$ post-surgery) as compared with vehicle-treated CCI rats (Fig. $2 \mathrm{C} ;{ }^{* * *} \mathrm{p}<0.001$ vs. Sham, ${ }^{*} \mathrm{p}<0.05,{ }^{\# \#} \mathrm{p}<0.01,{ }^{\# \# *} \mathrm{p}<0.001$ vs. vehicletreated group). In addition, the AUC (\%) data analysis showed a significant analgesic effect of L-NIL on the development of thermal hyperalgesia in neuropathic rats (Fig. $2 \mathrm{D} ;{ }^{* * *} \mathrm{p}<0.001$ vs. Sham, $\#$ \# $<0.001$ vs. vehicle-treated group).

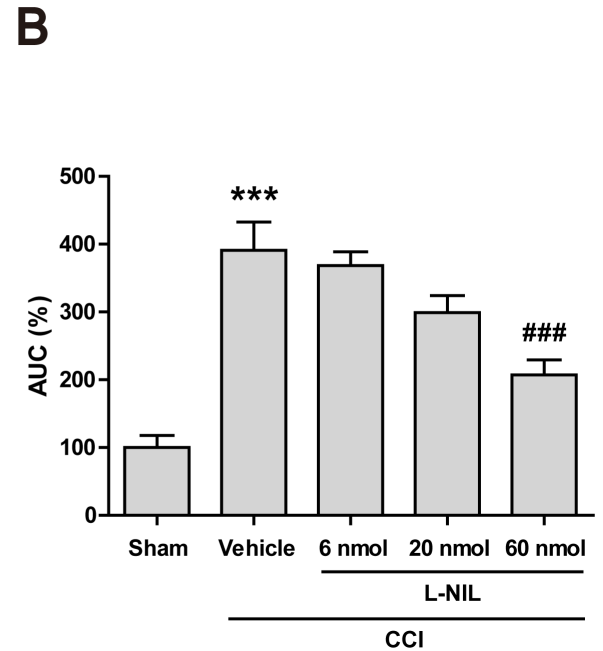

D

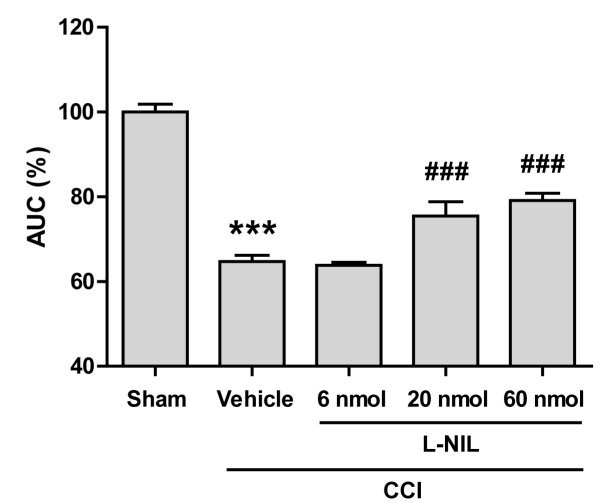

Fig. 2. Graphs illustrating the effect of i.t. administration of the NOS-II inhibitor, L-NIL on the development of neuropathic pain in CCI rats. (A and B) Paw withdrawal frequency (PWF, \%) was measured in the hind paw using a von-Frey filament $(2.0 \mathrm{~g})$. Sciatic nerve injury increased PWF, and this increase was dose-dependently suppressed by intrathecal (i.t.) administration of L-NIL (A; 6, 20 or $60 \mathrm{nmol}$ ). The area under curve (AUC, \%) data analysis showed an analgesic effect of L-NIL on CCI-induced mechanical allodynia (B). (C and D) Paw withdrawal latency (PWL, s) was measured in the hind paw using a plantar analgesia meter. Sciatic nerve injury decreased PWL, and this decrease was dose-dependently suppressed by i.t. administration of LNIL (B; 6, 20 or $60 \mathrm{nmol}$ ). The AUC (\%) data analysis showed an analgesic effect of L-NIL on CCI-induced thermal hyperalgesia (D). Drug or vehicle was administrated twice a day from days 0 to 5 post-surgery. $\mathrm{n}=6$ rats/group. ${ }^{* * *} \mathrm{p}<0.001$ vs. Sham; ${ }^{*} \mathrm{p}<0.05,{ }^{\# *} \mathrm{p}<0.01,{ }^{* \# *} \mathrm{p}<0.001$ vs. vehicle-treated group. A and C, two-way ANOVA followed by a Bonferroni multiple comparison test for post-hoc analysis. B and D, one-way ANOVA followed by a NewmanKeuls multiple comparison test for post-hoc analysis. 
I.t. administration of L-NIL suppresses the CCI-induced increase in PKC- and PKA-dependent GluN1 phosphorylation in the lumbar spinal cord dorsal horn of rats

In order to determine whether NOS-II modulates the functional activation of NMDA receptors, we examined the changes in the PKC- and PKA-dependent phosphorylation of the NMDA receptor GluN1 subunit (pGluN1) using Western blot analysis. Sciatic nerve injury increased PKC-dependent pGluN1 at the Ser896 site (Fig. 3A) and PKA-dependent pGluN1 at the Ser897 site (Fig. $3 \mathrm{~B})$ in the lumbar spinal cord dorsal horn at day 5 post-surgery as compared to that of the sham group (Fig. 3 ; ${ }^{\star} \mathrm{p}<0.05$ vs. Sham). Intrathecal administration of L-NIL (60 nmol) during the induction phase of neuropathic pain (from days $0 \sim 5$ post-surgery) significantly decreased not only the PKC-dependent pGluN1 at the

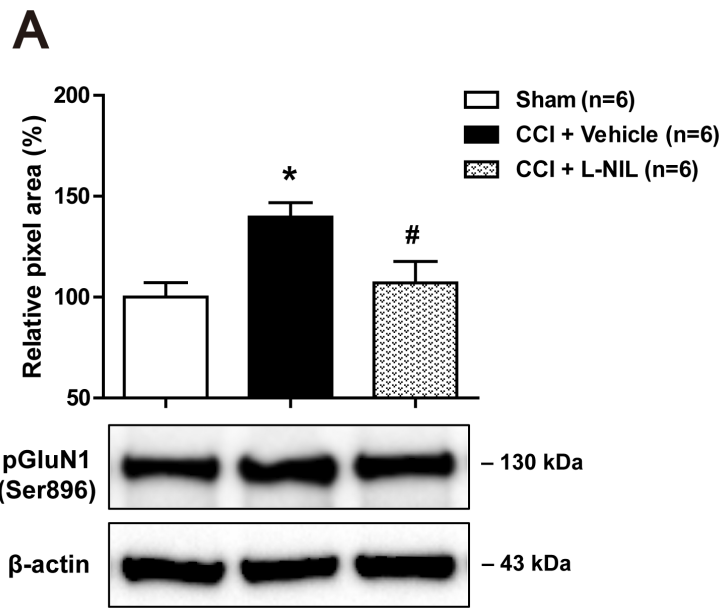

B

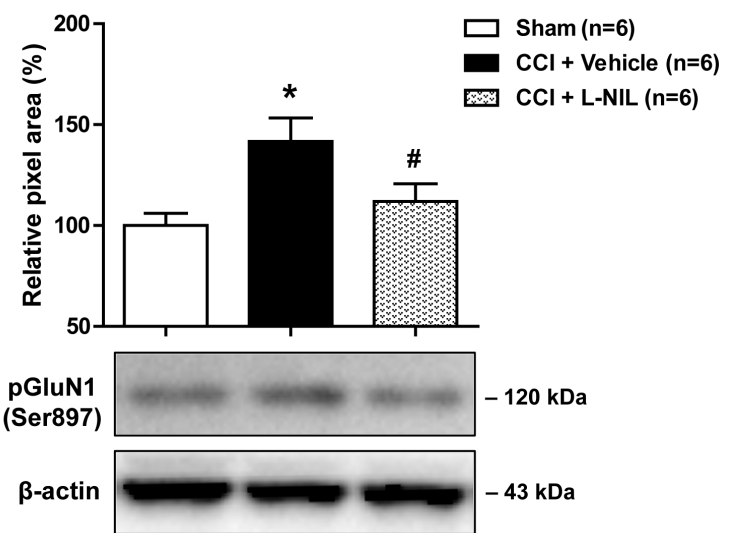

Fig. 3. Graphs illustrating the effect of i.t. administration of the NOS-II inhibitor, L-NIL on PKC- and PKA-dependent GluN1 phosphorylation in the lumbar spinal cord dorsal horn of CCI rats. (A and B) Results of Western blot analysis showed that the CCI-induced increase in PKC-dependent GluN1 phosphorylation (pGluN1) at the Ser896 site (A) and PKA-dependent pGluN1 at the Ser897 site in the spinal cord was significantly reduced by i.t. administration of L-NIL $(60 \mathrm{nmol})$. The drug or vehicle was administrated twice a day from days 0 to 5 post-surgery. The spinal cord dorsal horn was sampled at 5 days post-surgery. $n=6$ rats/group. ${ }^{*} \mathrm{p}<0.05$ vs. Sham; ${ }^{*} \mathrm{p}<0.05$ vs. vehicle-treated group. A and B, one-way ANOVA followed by a NewmanKeuls multiple comparison test for post-hoc analysis.

A

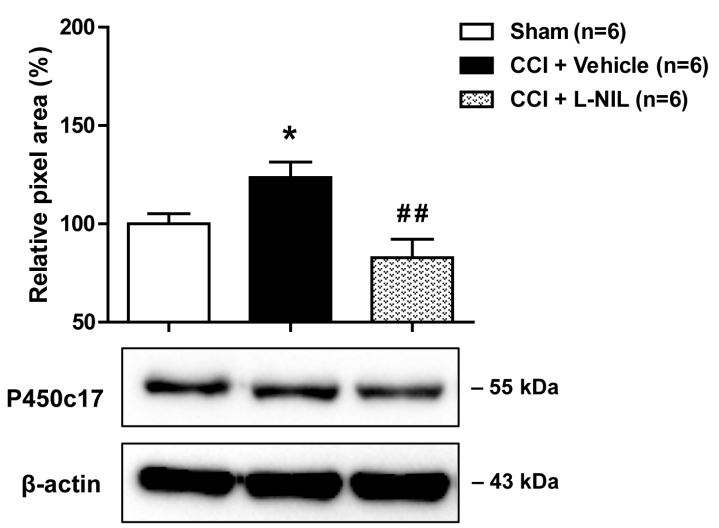

B

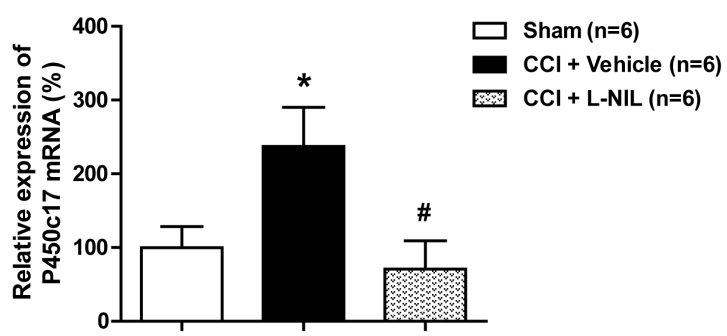

Fig. 4. Graphs illustrating the effect of i.t. administration of the NOS-II inhibitor, L-NIL on the protein and mRNA levels of P450c17 in the lumbar spinal cord dorsal horn of CCI rats. (A) Results of Western blot analysis showed that the CCI-induced increase in the protein level of cytochrome P450c17 in the spinal cord was suppressed by intrathecal (i.t.) administration of L-NIL ( $60 \mathrm{nmol})$. $\mathrm{n}=6 \mathrm{rats} /$ group. (B) The CCI-induced increase in the level of P450c17 mRNA in the spinal cord was also suppressed by i.t. administration of L-NIL ( $60 \mathrm{nmol})$. $\mathrm{n}=6 \mathrm{rats} /$ group. Drug or vehicle was administrated twice a day from days 0 to 5 post-surgery. The spinal cord dorsal horn was sampled at 5 days after surgery. ${ }^{*} \mathrm{p}<0.05 \mathrm{vs}$. Sham; ${ }^{*} \mathrm{p}<0.05$, ${ }^{\sharp *} \mathrm{p}<0.01 \mathrm{vs}$. vehicletreated group. A and B, one-way ANOVA followed by a Newman-Keuls multiple comparison test for post-hoc analysis. 
Ser896 site (Fig. 3A) but also the PKA-dependent pGluN1 at the Ser897 site (Fig. 3B) in CCI rats (Fig. 3; ${ }^{*} \mathrm{p}<0.05$ vs. vehicle-treated group). These results demonstrate that activation of NOS-II increases the PKC- and PKA-dependent phosphorylation of GluN1 in the lumbar spinal cord dorsal horn, which may lead to the potentiation of NMDA receptor function and the development of neuropathic pain following CCI.
I.t. administration of L-NIL suppresses the increases in protein and mRNA levels of P450c17 in the lumbar spinal cord dorsal horn of CCI rats

Next, we examined the effect of the NOS-II inhibitor, L-NIL on the protein and mRNA levels of the key DHEA-synthesizing enzyme, cytochrome P450c17 in the lumbar spinal cord dorsal horn using Western blot analysis and quantitative real-time PCR, respectively. Sciatic nerve injury significantly increased the protein level of P450c17 in the lumbar spinal cord dorsal horn at day 5 post-surgery as compared to that of the sham group (Fig. 4A;

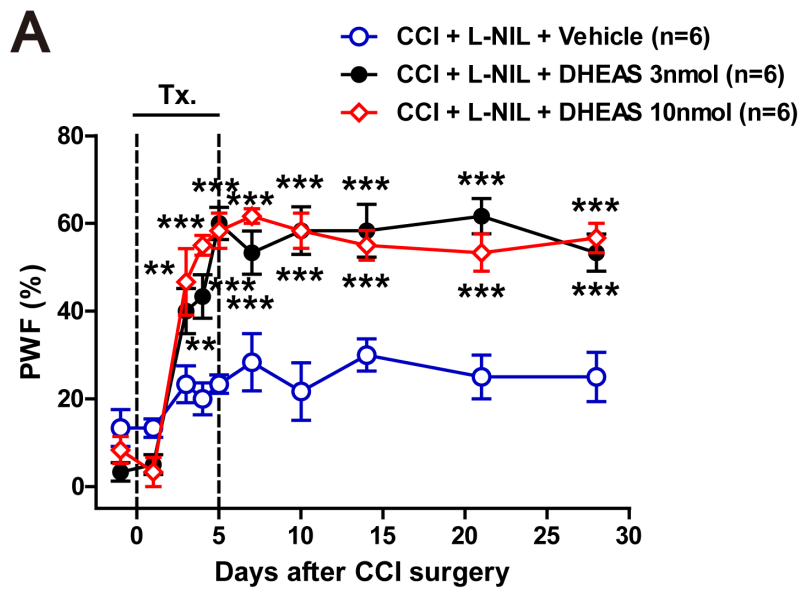

B
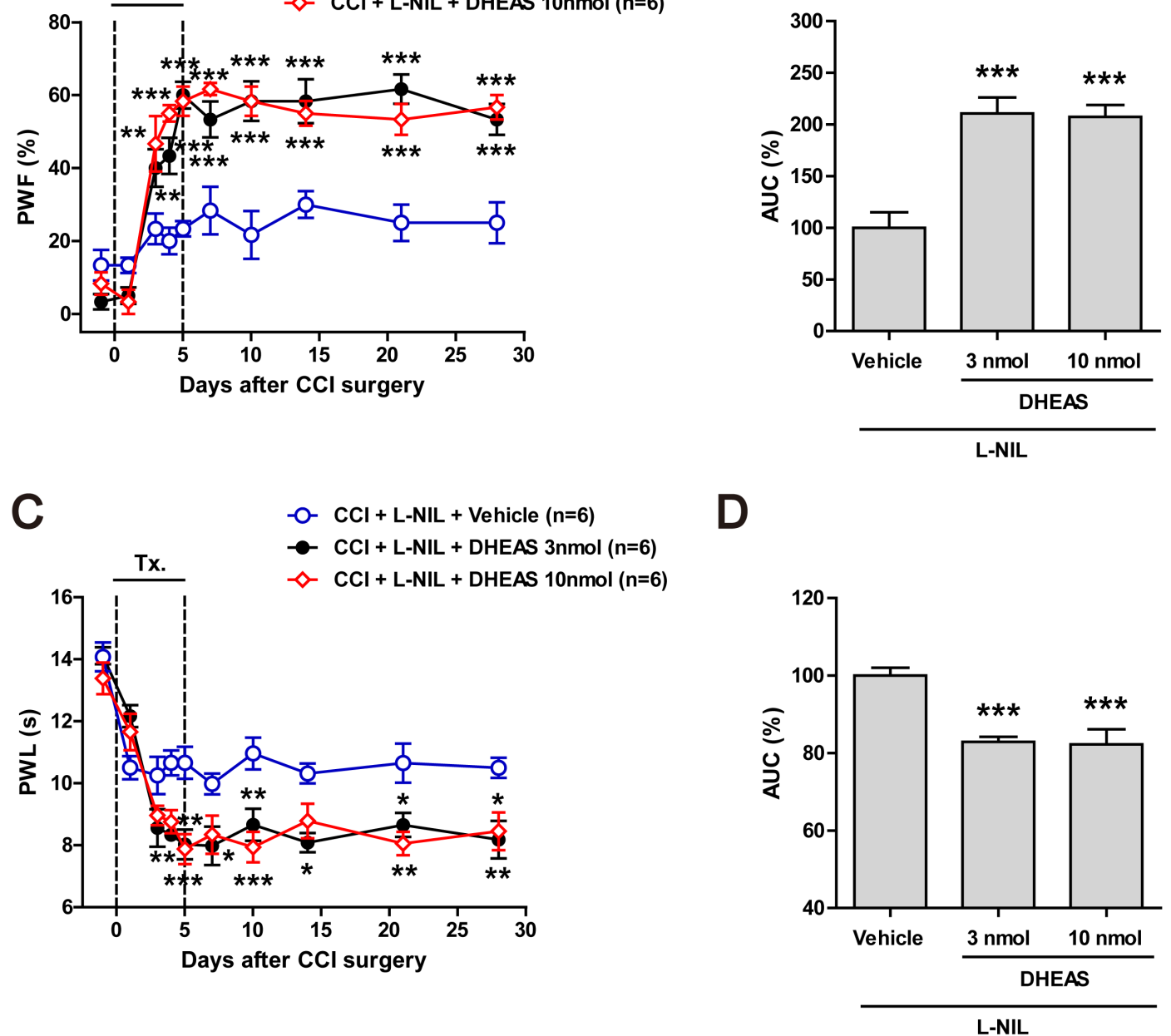

Fig. 5. Graphs illustrating the effect of DHEAS on the development of neuropathic pain originally inhibited by i.t. administration of L-NIL in CCI rats. (A and B) Paw withdrawal frequency (PWF, \%) was measured in the hind paw using a von-Frey filament (2.0 g). Intrathecal (i.t.) co-administration of dehydroepiandrosterone sulfate (DHEAS; 3 or $10 \mathrm{nmol}$ ) with L-NIL (60 nmol) restored the PWF (A) and the area under curve (AUC, \%) data (B) that were inhibited by administration of L-NIL $(60 \mathrm{nmol}$ ) alone. (C and D) Paw withdrawal latency (PWL, s) was measured in the hind paw using a plantar analgesia meter. Co-administration of DHEAS (3 or $10 \mathrm{nmol})$ with L-NIL (60 nmol) restored the PWL (C) and the AUC (\%) data (D) that were originally inhibited by administration of L-NIL ( $60 \mathrm{nmol})$ alone. Drugs or vehicle were administrated twice a day from days 0 to 5 post-surgery. $\mathrm{n}=6$ rats/ group. ${ }^{*} \mathrm{p}<0.05,{ }^{* *} \mathrm{p}<0.01,{ }^{* *} \mathrm{p}<0.001$ vs. L-NIL-treated group. A and C, two-way ANOVA followed by a Bonferroni multiple comparison test for posthoc analysis. B and D, one-way ANOVA followed by a Newman-Keuls multiple comparison test for post-hoc analysis. 
${ }^{*} \mathrm{p}<0.05 \mathrm{vs}$. Sham), and this increase was significantly inhibited by i.t. administration of L-NIL ( $60 \mathrm{nmol}$ ) during the induction phase of neuropathic pain (from days $0 \sim 5$ post-surgery) in CCI rats (Fig.

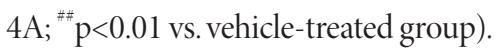

In addition, sciatic nerve injury significantly increased the mRNA level of P450c17 in the lumbar spinal cord dorsal horn at day 5 post-surgery as compared to that of the sham group (Fig. 4B; ${ }^{*} \mathrm{p}<0.05$ vs. Sham), and this increase was significantly inhibited by i.t. administration of L-NIL ( $60 \mathrm{nmol}$ ) during the induction phase of neuropathic pain (from days $0 \sim 5$ post-surgery) in CCI rats (Fig. $4 \mathrm{~B} ;{ }^{\sharp} \mathrm{p}<0.05$ vs. vehicle-treated group). These results demonstrate that activation of NOS-II increases the protein and mRNA levels of the steroidogenic enzyme P450c17 in the lumbar spinal cord dorsal horn of CCI rats.

\section{Co-administration of DHEAS restores the CCI-induced de- velopment of neuropathic pain that was suppressed by $L$ - NIL administration alone}

To determine whether spinal P450c17, a DHEA-synthesizing enzyme, contributes to the NOS-II-induced development of neuropathic pain in CCI rats, dehydroepiandrosterone sulfate (DHEAS) was co-administrated with L-NIL on postoperative days $0 \sim 5$. Since DHEAS is more stable than DHEA, DHEAS was used in the present study. Repeated daily intrathecal co-administration of DHEAS
( 3 or $10 \mathrm{nmol}$ ) with L-NIL (60 nmol) during the induction phase of neuropathic pain restored the CCI-induced development of mechanical allodynia that was originally inhibited by L-NIL administration alone (Fig. 5A; ${ }^{* *} \mathrm{p}<0.01,{ }^{* * *} \mathrm{p}<0.001$ vs. L-NIL-treated group). In addition, the AUC (\%) data analysis showed that the LNIL-induced analgesic effect on the development of mechanical allodynia was restored by co-administration of DHEAS with LNIL in neuropathic rats (Fig. $5 B ;{ }^{* * *} \mathrm{p}<0.001$ vs. L-NIL-treated group).

Moreover, i.t. co-administration of DHEAS (3 or $10 \mathrm{nmol}$ ) and L-NIL (60 nmol) during the induction phase of neuropathic pain restored the CCI-induced development of thermal hyperalgesia that was originally inhibited by L-NIL administration alone (Fig. $5 \mathrm{C} ;{ }^{*} \mathrm{p}<0.05,{ }^{* *} \mathrm{p}<0.01,{ }^{* *} \mathrm{p}<0.001$ vs. L-NIL-treated group). In addition, the AUC (\%) data analysis showed that the L-NIL-induced analgesic effect on the development of thermal hyperalgesia was restored by co-administration of DHEAS with L-NIL in neuropathic rats (Fig. $5 \mathrm{D} ;{ }^{* * *} \mathrm{p}<0.001$ vs. L-NIL-treated group).

\section{Co-administration of DHEAS restores the CCI-induced increase in PKC- and PKA-dependent GluN1 phosphoryla- tion that were suppressed by L-NIL administration alone}

In order to determine whether spinal P450c17 plays a role in the NOS-II-induced increase in the functional potentiation of NMDA
A

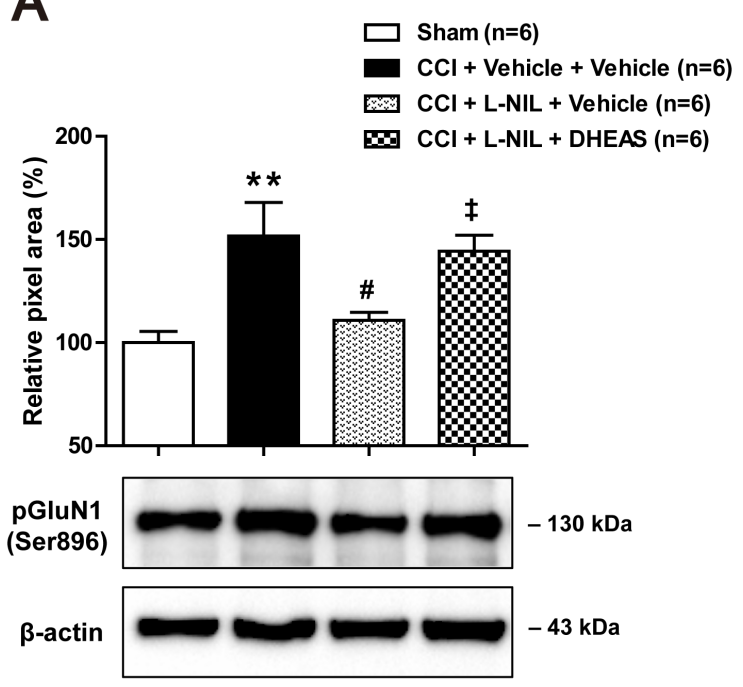

B
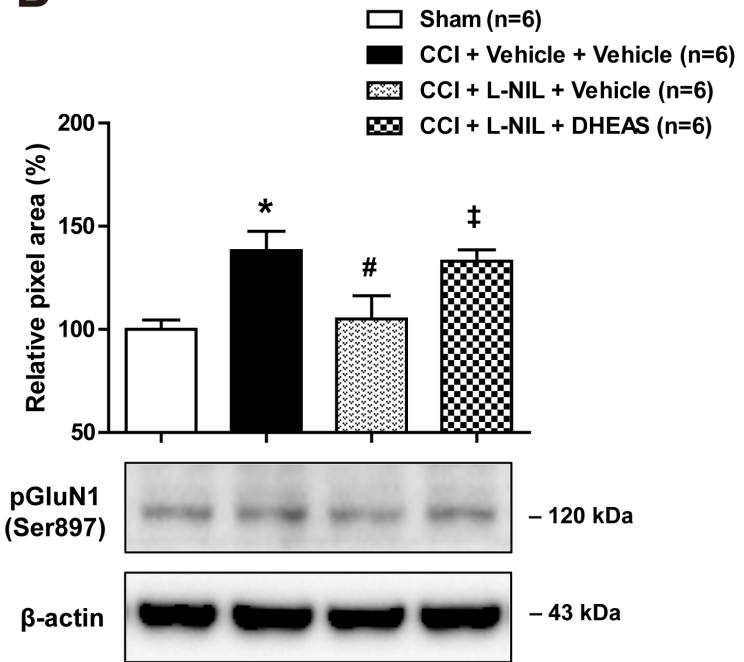

Fig. 6. Graphs illustrating the effect of DHEAS on the PKC- and PKA-dependent GluN1 phosphorylation originally inhibited by i.t. administration of L-NIL in CCI rats. (A and B) Results of Western blot analysis showing that co-administration of dehydroepiandrosterone sulfate (DHEAS; 10 nmol) with L-NIL (60 nmol) restored the CCI-induced increase in PKC-dependent GluN1 phosphorylation (pGluN1) at the Ser896 site (A) and PKAdependent pGluN1 at the Ser897 site (B) in the spinal cord that were initially inhibited by administration of L-NIL (60 nmol) alone. Drugs or vehicle were administrated twice a day from days 0 to 5 post-surgery. The spinal cord dorsal horn was sampled at 5 days post-surgery. $n=6$ rats/group. ${ }^{*} \mathrm{p}<0.05$, ${ }^{* *} \mathrm{p}<0.01$ vs. Sham; ${ }^{*} \mathrm{p}<0.05$ vs. vehicle-treated group; ${ }^{*} \mathrm{p}<0.05$ vs. L-NIL-treated group. A and B, one-way ANOVA followed by a Newman-Keuls multiple comparison test for post-hoc analysis. 
receptors, DHEAS was co-administrated with L-NIL on postoperative days $0 \sim 5$ and the changes in the PKC- and PKA-dependent GluN1 phosphorylation was then examined using Western blot analysis. Repeated daily intrathecal co-administration of DHEAS $(10 \mathrm{nmol})$ with L-NIL $(60 \mathrm{nmol})$ during the induction phase of neuropathic pain restored the CCI-induced increase in the PKCdependent GluN1 phosphorylation at the Ser896 site (Fig. 6A) and the PKA-dependent GluN1 phosphorylation at the Ser897 site (Fig. $6 \mathrm{~B})$ in the lumbar spinal cord dorsal horn at day 5 post-surgery as compared to that of the L-NIL-treated group (Fig. 6 ; ${ }^{*} \mathrm{p}<0.05$, ${ }^{* *} \mathrm{p}<0.01$ vs. Sham, ${ }^{*} \mathrm{p}<0.05$ vs. vehicle-treated group, ${ }^{*} \mathrm{p}<0.05$ vs. L-NIL-treated group). These results demonstrate that spinal P450c17, a DHEA-synthesizing enzyme, plays an important role in the NOS-II-induced potentiation of NMDA receptor function, as well as, the development of neuropathic pain in CCI rats.

\section{DISCUSSION}

The processing of inflammatory and neuropathic pain is associated with the production of $\mathrm{NO}$ in the spinal cord. In the present study, sciatic nerve injury induced a significant increase in the expression of NOS-II in spinal cord microglial cells and in the total NO levels in the lumbar spinal cord dorsal horn at day 5 post-surgery. Inhibition of NOS-II with L-NIL during the induction phase of neuropathic pain (from days 0 to 5 post-surgery) significantly reduced the development of mechanical allodynia and thermal hyperalgesia as well as the CCI-induced increase in PKC- and PKA-dependent pGluN1 expression. In addition, sciatic nerve injury increased the protein and mRNA levels of cytochrome P450c17, a DHEA-synthesizing enzyme, in the lumbar spinal cord dorsal horn at day 5 post-surgery, and this increase was suppressed by repeated i.t. administration of L-NIL. Furthermore, i.t. administration of the sulfated form of DHEA, DHEAS restored the CCI-induced development of mechanical allodynia and thermal hyperalgesia, as well as, increased pGluN1 expression, which were originally attenuated by i.t. administration of L-NIL alone. Collectively these findings suggest that activation of NOS-II increases the expression of spinal P450c17, ultimately contributing to the increase in phosphorylation of NMDA receptor GluN1 subunit and the development of neuropathic pain associated with peripheral nerve injury.

Nitric oxide plays an important role in various physiological and pathological processes in the nervous system. At low concentrations, NO plays a role in neurotransmission and as a neuromodulator serving to regulate aspects of nervous system function, while at higher concentrations, $\mathrm{NO}$ increases oxidative stress and has a role in the pathogenesis of inflammatory and neuropathic pain
$[16,21]$. Nitric oxide synthase type II is an inducible NOS that is expressed in glial cells of the central nervous system by a wide variety of pro-inflammatory stimuli [22-24]. In vitro, inflammatory cytokines such as TNFa, IL-1 and IFN $\gamma$, which are also upregulated following nerve injury in vivo, can synergize in stimulated glial cells to express other cytotoxic inflammatory mediators such as NOS-II and to release high titers of $\mathrm{NO}[25,26]$. In several types of nerve injury, mice lacking NOS-II show evidence of a myelinated fiber Wallerian degeneration delay, which is associated with delayed expression of neuropathic pain [27]. In the present study, sciatic nerve injury increased the expression of NOS-II in microglial cells and concomitant $\mathrm{NO}$ production in the spinal cord of neuropathic rats. In addition, inhibition of NOS-II during the induction phase of neuropathic pain significantly suppressed the development of neuropathic pain. These results suggest the possibility that microglial NOS-II-induced initiation of a neuroinflammatory process plays a critical role in the pathological changes in the spinal cord under peripheral nerve injury-associated neuropathic pain conditions.

Neurosteroids are locally synthesized in the central nervous system following nerve injuries as independent from steroidogenic organs [28-30]. Among steroidogenic enzymes, cytochrome P450c17 catalyzes the 17-alpha-hydroxylation of pregnenolone and subsequent cleavage of the residual two carbons at $\mathrm{C} 17$ leading to the formation of DHEA. DHEA has an effect on the spinal nociceptive signal transmission producing a rapid pronociceptive effect [31], and its sulfate ester DHEAS also plays an important role in the enhancement of NMDA-induced nociception via the activation of spinal sigma-1 receptors, but not through the inhibition of $\mathrm{GABA}_{\mathrm{A}}$ receptors [8]. In a previous study from our laboratories, $\mathrm{P} 450 \mathrm{c} 17$ immunostaining was shown to increase in spinal astrocytes and inhibition of P450c17 significantly decreased the activity of astrocyte sigma-1 receptors in CCI mice [9]. In the present study, inhibition of NOS-II with L-NIL significantly reduced not only the protein expression of P450c17, but also the mRNA levels of P450c17 in the lumbar spinal cord dorsal horn of CCI rats. In addition, DHEAS administration restored the development of neuropathic pain that was originally suppressed by L-NIL administration alone. The current results suggest the possibility that NOS-II could change the neurosteroid-mediated synaptic microenvironment through modulation of astrocyte P450c17 expression at the gene transcription level, ultimately contributing to spinal nociceptive signaling during the induction phase of neuropathic pain in CCI rats.

Regulation of gene transcription in response to extracellular signals is initiated by receptor molecules and mediated by a broad spectrum of intracellular signaling cascades [14]. It has been sug- 
gested that diverse ion channels, protein tyrosine kinases, mitogenactivated protein kinases and protein phosphatases can be the targets of $\mathrm{NO}$ [14]. In addition, $\mathrm{NO}$ has other molecular targets resulting in regulation of diverse gene expression. NO does not only directly influence the activity of transcription factors including nuclear factor (NF)- $\kappa \mathrm{B}$, activating protein (AP)- 1 and specificity protein 1 (Sp1), but it also modulates mRNA stability and translation, as well as, the processing of the primary gene products [14]. It has been demonstrated that the transcription of the $\mathrm{P} 450 \mathrm{c} 17$ gene is regulated by cAMP-dependent binding of steroidogenic factor- 1 (SF-1) to its promoter in the adrenal cortex [32]. Furthermore, the nuclear phosphoprotein SET binds to the specific DNA site of the rat $\mathrm{P} 450 \mathrm{c} 17$ gene and transactivates $\mathrm{P} 450 \mathrm{c} 17$ in neuronal cells [33]. There is the possibility that NO regulates the expression of the $\mathrm{P} 450 \mathrm{c} 17$ gene through the modulation of transcription or posttranscription, however, the detailed mechanisms by which spinal NOS-II/NO signaling regulates the expression of $\mathrm{P} 450 \mathrm{cl} 7$ need to be further investigated in future studies.

In the present study, we focused our investigation on the NOSII-induced positive modulation of $\mathrm{P} 450 \mathrm{c} 17$ expression in the spinal cord as a potential nociceptive mechanism that underlies the development of neuropathic pain following peripheral nerve injury in rats. By contrast, studies in multiple species have demonstrated primarily an inhibition of steroidogenesis by $\mathrm{NO}$ in endocrine tissues $[34,35]$. These actions are supported by an in vivo study showing that the stress-induced down-regulation of testicular steroidogenesis is mediated by the intratesticular $\mathrm{NO}$ signaling pathway [34]. In the ovary, NO regulation of blood flow may provide a key means of altering steroid formation, while in the adrenal gland, NO may alter steroid production by acting directly on the steroidogenic enzymes themselves or by altering their expression via effects on transcription factor function [35]. Thus, these results suggest that the underlying mechanisms in NOinduced regulation of steroidogenesis may depend on the tissue types and the environment status. Unlike the inhibitory effects that occur with NO in steroidogenic tissues, increased NO signaling in spinal cord appears to contribute to the neurosteroidogenesis induced by $\mathrm{P} 450 \mathrm{c} 17$ and nociceptive signaling transmission under pathological conditions such as neuropathic pain. In this regard, it is likely that NO modulates neurosteroidogenesis in the spinal cord in association with nociception in different ways than those associated with other types of NO-related signaling that occur in steroidogenic organs.

In the spinal cord, the activation of NMDA receptors is known to be an essential contributor to the process of 'central sensitization', a phenomenon in which nociceptive inputs to the dorsal horn increase the excitability and synaptic efficacy of neurons in spinal pain pathways [36]. It has been suggested that selective inhibition of NOS-II blocks the increased responses of rat dorsal horn neurons, as well as, the development of secondary hyperalgesia following intradermal capsaicin injection representing a spinal contribution to central sensitization [37]. This is consistent with the results of the present study showing that inhibition of NOS-II during the induction phase of neuropathic pain significantly decreases the PKC- and PKA-dependent phosphorylation of the NMDA receptor GluN1 subunit, and importantly this decrease is restored by co-administration of DHEAS with L-NIL. These results suggest a potential role of NOS-II in the early phosphorylation-dependent phase of central sensitization, which is possibly mediated by DHEAS induced by P450c17. Moreover, it has been demonstrated that PKC modulates functional NMDA receptor channel trafficking to the plasma membrane as well as increases the probability of channel openings [38, 39]. Thus, the spinal NOS-II-induced increase in GluN1 phosphorylation could play a critical role in the functional activation of NMDA receptors and the development of central sensitization during the early phase of neuropathic pain.

In conclusion, the present study demonstrates that activation of NOS-II increases the expression of the DHEA-synthesizing enzyme, $\mathrm{P} 450 \mathrm{c} 17$ in the lumbar spinal cord dorsal horn during the induction phase of neuropathic pain. Moreover, the sulfated form of DHEA, DHEAS also appears to mediate NOS-II's effects on PKC- and PKA-dependent phosphorylation of the NMDA receptor GluN1 subunit and the development of CCI-induced neuropathic pain. Collectively these findings suggest that the NOS-IIinduced increase in $\mathrm{P} 450 \mathrm{c} 17$ plays a critical role in spinal NOSII-induced central sensitization, and ultimately contributes to the development of neuropathic pain associated with peripheral nerve injury.

\section{ACKNOWLEDGEMENTS}

This research was supported by the National Research Foundation of Korea (NRF) grant (No. 2017R1A2A2A05001402) funded by the Korean Government, South Korea.

\section{REFERENCES}

1. Baulieu EE (1997) Neurosteroids: of the nervous system, by the nervous system, for the nervous system. Recent Prog Horm Res 52:1-32.

2. Compagnone NA, Mellon SH (2000) Neurosteroids: biosynthesis and function of these novel neuromodulators. Front Neuroendocrinol 21:1-56.

3. Corpéchot C, Robel P, Axelson M, Sjövall J, Baulieu EE (1981) 
Characterization and measurement of dehydroepiandrosterone sulfate in rat brain. Proc Natl Acad Sci U S A 78:47044707.

4. Baulieu EE, Robel P (1998) Dehydroepiandrosterone (DHEA) and dehydroepiandrosterone sulfate (DHEAS) as neuroactive neurosteroids. Proc Natl Acad Sci U S A 95:40894091.

5. Monnet FP, Maurice T (2006) The sigmal protein as a target for the non-genomic effects of neuro(active)steroids: molecular, physiological, and behavioral aspects. J Pharmacol Sci 100:93-118.

6. Meyer JH, Gruol DL (1994) Dehydroepiandrosterone sulfate alters synaptic potentials in area CA1 of the hippocampal slice. Brain Res 633:253-261.

7. Vallée M, Mayo W, Le Moal M (2001) Role of pregnenolone, dehydroepiandrosterone and their sulfate esters on learning and memory in cognitive aging. Brain Res Brain Res Rev 37:301-312.

8. Yoon SY, Roh DH, Seo HS, Kang SY, Moon JY, Song S, Beitz AJ, Lee JH (2010) An increase in spinal dehydroepiandrosterone sulfate (DHEAS) enhances NMDA-induced pain via phosphorylation of the NR1 subunit in mice: involvement of the sigma-1 receptor. Neuropharmacology 59:460-467.

9. Choi SR, Roh DH, Yoon SY, Choi HS, Kang SY, Han HJ, Beitz AJ, Lee JH (2019) Spinal cytochrome P450c17 plays a key role in the development of neuropathic mechanical allodynia: involvement of astrocyte sigma-1 receptors. Neuropharmacology 149:169-180.

10. Alderton WK, Cooper CE, Knowles RG (2001) Nitric oxide synthases: structure, function and inhibition. Biochem J 357:593-615.

11. Esplugues JV (2002) NO as a signalling molecule in the nervous system. Br J Pharmacol 135:1079-1095.

12. Cury Y, Picolo G, Gutierrez VP, Ferreira SH (2011) Pain and analgesia: the dual effect of nitric oxide in the nociceptive system. Nitric Oxide 25:243-254.

13. Meller ST, Gebhart GF (1993) Nitric oxide (NO) and nociceptive processing in the spinal cord. Pain 52:127-136.

14. Bogdan C (2001) Nitric oxide and the regulation of gene expression. Trends Cell Biol 11:66-75.

15. Levy D, Höke A, Zochodne DW (1999) Local expression of inducible nitric oxide synthase in an animal model of neuropathic pain. Neurosci Lett 260:207-209.

16. De Alba J, Clayton NM, Collins SD, Colthup P, Chessell I, Knowles RG (2006) GW274150, a novel and highly selective inhibitor of the inducible isoform of nitric oxide synthase (iNOS), shows analgesic effects in rat models of inflamma- tory and neuropathic pain. Pain 120:170-181.

17. Bennett GJ, Xie YK (1988) A peripheral mononeuropathy in rat that produces disorders of pain sensation like those seen in man. Pain 33:87-107.

18. Choi SR, Roh DH, Yoon SY, Kang SY, Moon JY, Kwon SG, Choi HS, Han HJ, Beitz AJ, Oh SB, Lee JH (2013) Spinal sigma- 1 receptors activate NADPH oxidase 2 leading to the induction of pain hypersensitivity in mice and mechanical allodynia in neuropathic rats. Pharmacol Res 74:56-67.

19. Hargreaves K, Dubner R, Brown F, Flores C, Joris J (1988) A new and sensitive method for measuring thermal nociception in cutaneous hyperalgesia. Pain 32:77-88.

20. Choi SR, Kwon SG, Choi HS, Han HJ, Beitz AJ, Lee JH (2016) Neuronal NOS activates spinal NADPH oxidase 2 contributing to central sigma-1 receptor-induced pain hypersensitivity in mice. Biol Pharm Bull 39:1922-1931.

21. Conti A, Miscusi M, Cardali S, Germanò A, Suzuki H, Cuzzocrea S, Tomasello F (2007) Nitric oxide in the injured spinal cord: synthases cross-talk, oxidative stress and inflammation. Brain Res Brain Res Rev 54:205-218.

22. Saha RN, Pahan K (2006) Regulation of inducible nitric oxide synthase gene in glial cells. Antioxid Redox Signal 8:929-947.

23. Galea E, Feinstein DL, Reis DJ (1992) Induction of calciumindependent nitric oxide synthase activity in primary rat glial cultures. Proc Natl Acad Sci U S A 89:10945-10949.

24. Nomura Y, Kitamura Y (1993) Inducible nitric oxide synthase in glial cells. Neurosci Res 18:103-107.

25. Tran EH, Hardin-Pouzet H, Verge G, Owens T (1997) Astrocytes and microglia express inducible nitric oxide synthase in mice with experimental allergic encephalomyelitis. J Neuroimmunol 74:121-129.

26. Jeong HK, Ji K, Min K, Joe EH (2013) Brain inflammation and microglia: facts and misconceptions. Exp Neurobiol 22:59-67.

27. Levy D, Kubes P, Zochodne DW (2001) Delayed peripheral nerve degeneration, regeneration, and pain in mice lacking inducible nitric oxide synthase. J Neuropathol Exp Neurol 60:411-421.

28. García-Estrada J, Luquín S, Fernández AM, Garcia-Segura LM (1999) Dehydroepiandrosterone, pregnenolone and sex steroids down-regulate reactive astroglia in the male rat brain after a penetrating brain injury. Int J Dev Neurosci 17:145151.

29. Patte-Mensah C, Mensah-Nyagan AG (2008) Peripheral neuropathy and neurosteroid formation in the central nervous system. Brain Res Brain Res Rev 57:454-459.

30. Kibaly C, Patte-Mensah C, Mensah-Nyagan AG (2005) Mo- 
lecular and neurochemical evidence for the biosynthesis of dehydroepiandrosterone in the adult rat spinal cord. J Neurochem 93:1220-1230.

31. Kibaly C, Meyer L, Patte-Mensah C, Mensah-Nyagan AG (2008) Biochemical and functional evidence for the control of pain mechanisms by dehydroepiandrosterone endogenously synthesized in the spinal cord. FASEB J 22:93-104.

32. Dammer EB, Leon A, Sewer MB (2007) Coregulator exchange and sphingosine-sensitive cooperativity of steroidogenic factor-1, general control nonderepressed 5, p54, and p160 coactivators regulate cyclic adenosine 3',5'-monophosphate-dependent cytochrome $\mathrm{P} 450 \mathrm{c} 17$ transcription rate. Mol Endocrinol 21:415-438.

33. Compagnone NA, Zhang P, Vigne JL, Mellon SH (2000) Novel role for the nuclear phosphoprotein SET in transcriptional activation of $\mathrm{P} 450 \mathrm{c} 17$ and initiation of neurosteroidogenesis. Mol Endocrinol 14:875-888.

34. Kostic TS, Andric SA, Maric D, Stojilkovic SS, Kovacevic $R$ (1999) Involvement of inducible nitric oxide synthase in stress-impaired testicular steroidogenesis. J Endocrinol 163:409-416.

35. Ducsay CA, Myers DA (2011) eNOS activation and NO function: differential control of steroidogenesis by nitric oxide and its adaptation with hypoxia. J Endocrinol 210:259-269.

36. Woolf CJ, Mannion RJ (1999) Neuropathic pain: aetiology, symptoms, mechanisms, and management. Lancet 353:19591964.

37. Wu J, Fang L, Lin Q, Willis WD (2001) Nitric oxide synthase in spinal cord central sensitization following intradermal injection of capsaicin. Pain 94:47-58.

38. Chen L, Huang LY (1992) Protein kinase C reduces Mg2+ block of NMDA-receptor channels as a mechanism of modulation. Nature 356:521-523.

39. Lan JY, Skeberdis VA, Jover T, Grooms SY, Lin Y, Araneda RC, Zheng X, Bennett MV, Zukin RS (2001) Protein kinase C modulates NMDA receptor trafficking and gating. Nat Neurosci 4:382-390. 